\title{
The angular power spectrum measurement of the Galactic synchrotron emission using the TGSS survey
}

\author{
Samir Choudhuri ${ }^{1,2}$, Somnath Bharadwaj ${ }^{2}$, Sk. Saiyad Ali ${ }^{3}$, \\ Nirupam Roy ${ }^{4}$, H. T. Intema ${ }^{5}$ and Abhik Ghosh ${ }^{6,7}$ \\ ${ }^{1}$ National Centre For Radio Astrophysics, Post Bag 3, Ganeshkhind, Pune 411 007, India \\ ${ }^{2}$ Department of Physics, \& Centre for Theoretical Studies, IIT Kharagpur, Kharagpur 721 \\ 302, India \\ ${ }^{3}$ Department of Physics,Jadavpur University, Kolkata 700032, India \\ ${ }^{4}$ Department of Physics, Indian Institute of Science, Bangalore 560012, India \\ ${ }^{5}$ Leiden Observatory, Leiden University, Niels Bohrweg 2, NL-2333CA, Leiden, The \\ Netherlands \\ ${ }^{6}$ Department of Physics and Astronomy, University of the Western Cape, Robert Sobukwe \\ Road, Bellville 7535, South Africa \\ ${ }^{7}$ SKA SA, The Park, Park Road, Pinelands 7405, South Africa
}

\begin{abstract}
Characterizing the diffuse Galactic synchrotron emission (DGSE) at arcminute angular scales is needed to remove this foregrounds in cosmological $21-\mathrm{cm}$ measurements. Here, we present the angular power spectrum $\left(C_{\ell}\right)$ measurement of the diffuse Galactic synchrotron emission using two fields observed by the TIFR GMRT Sky Survey (TGSS). We apply 2D Tapered Gridded Estimator (TGE) to estimate the $C_{\ell}$ from the visibilities. We find that the residual data after subtracting the point sources is likely dominated by the diffuse Galactic synchrotron radiation across the angular multipole range $240 \leqslant \ell \lesssim 500$. We fit a power law to the measured $C_{\ell}$ over this $\ell$ range. We find that the slopes in both fields are consistent with earlier measurements. For the second field, however, we interpret the measured $C_{\ell}$ as an upper limit for the DGSE as there is an indication of a significant residual point source contribution.
\end{abstract}

Keywords. methods: statistical, data analysis - techniques: interferometric- cosmology: diffuse radiation

\section{Introduction}

Observations of the redshifted neutral hydrogen (HI) 21-cm radiation can be used to probe a wide range of cosmological and astrophysical phenomena over a large redshift range $0<z \lesssim 200$ (Bharadwaj \& Ali 2005; Pritchard \& Loeb 2012; Mellema et al. 2013). There are several ongoing and future experiments such as the Donald C. Backer Precision Array to Probe the Epoch of Reionization (PAPER,Parsons et al. 2010), the Low Frequency Array (LOFAR, van Haarlem et al. 2013; Yatawatta, et al. 2013) and the Murchison Wide-field Array (MWA, Bowman et al. 2013; Tingay et al. 2013), the Square Kilometer Array (SKA1 LOW, Koopmans et al. 2015) and the Hydrogen Epoch of Reionization Array (HERA, Neben, et al. 2016) which are aiming to detect the power spectrum of the 21 -cm signal from the Epoch of Reionization (EoR, $6 \lesssim z \lesssim 13$ ). The main challenges for detecting the cosmological $21-\mathrm{cm}$ signal are the astrophysical foregrounds which are 4-5 orders of magnitude brighter than the expected signal (Shaver et al. 1999; Santos, Cooray \& Knox 2005; Ali, Bharadwaj \& Chengalur 2008). The major foreground components include the point sources, diffuse Galactic synchrotron emission, Galactic and extra-galactic free-free emission. The diffuse Galactic synchrotron emission 
(DGSE) is the most dominant foreground component at large angular scale. The detailed understanding of the DGSE is needed to remove this component in 21-cm experiments. The study of the DGSE also quantifies the fluctuations in the magnetic field and in the electron density of the turbulent interstellar medium (ISM) of our Galaxy (e.g. Waelkens, Schekochihin \& Enßlin 2009; Lazarian \& Pogosyan 2012; Iacobelli et al. 2013).

Several observations are made to characterize the DGSE at wide frequency range. Haslam et al. (1982) have quantified the all-sky diffuse Galactic synchrotron radiation at $408 \mathrm{MHz}$. Reich (1982) and Reich \& Reich (1988) have observed the Galactic synchrotron emission at higher frequency (1.4 GHz). Guzmán et al. (2011) have studied all-sky temperature variation at $45 \mathrm{MHz}$ using Maipu and Muradar array and also measured the spectral index by comparing this with Haslam et al. (1982) $408 \mathrm{MHz}$ map. Dowell et al. (2017) have presented the low-frequency all-sky map between $35 \mathrm{MHz}$ to $80 \mathrm{MHz}$. Bernardi et al. (2009) have estimated the $C_{\ell}$ using Westerbork Synthesis Radio Telescope (WSRT) observations and found that it follows a power law power spectrum up to multipole range $\ell \leqslant 900$. Ghosh et al. (2012) have analyzed GMRT $150 \mathrm{MHz}$ data and estimated the $C_{\ell}$ from the residual data. For both cases the slope of the measured $C_{\ell}$ is in the range $\beta=2$ to 3 . Recently, Iacobelli et al. (2013) reported that the $C_{\ell}$ of the foreground synchrotron fluctuations is approximately a power law with a slope $\beta \approx 1.8$ up to angular multipoles of 1300 .

Here, we present the $C_{\ell}$ measurement of the DGSE using two fields observed by the TIFR GMRT Sky Survey (TGSS†; Sirothia et al. 2014). We use the data from TGSS-ADR survey Intema et al. (2017) and apply the Tapered Gridded Estimator (TGE; Choudhuri et al. 2016) to the residual data to measure the $C_{\ell}$. We identify the angular multipole range where the measured $C_{\ell}$ is likely dominated by the DGSE, and we present power law fits in this region.

\section{Data Analysis}

We use two data sets from the TGSS survey and estimated the $C_{\ell}$ for them. The Galactic coordinates for these fields are $\left(9^{\circ},+10^{\circ}\right.$; Data1 $)$ and $\left(15^{\circ},-11^{\circ}\right.$; Data2). The central frequency of this survey is $147.5 \mathrm{MHz}$ with an instantaneous bandwidth of 16.7 MHz. These data sets were analysed with a fully automated pipeline based on the SPAM package (Intema et al. 2009; Intema 2014). The off source rms noise $\left(\sigma_{n}\right)$ for these fields are $4.1 \mathrm{mJy} /$ Beam and $3.1 \mathrm{mJy} /$ Beam for Data1 and Data2 respectively. We subtract all bright point sources from the central region of the telescope's primary beam to characterize the underlying diffuse emission.

We apply the TGE to estimate $C_{\ell}$ from the measured visibilities $\mathcal{V}_{i}$. The TGE incorporates three novel features. First, the estimator uses the gridded visibilities to make it computationally much faster. Second, a positive noise bias is removed by subtracting the auto-correlation of the visibilities. Third, the estimator allows us to taper the primary beam so as to restrict the contribution from the sources in the outer regions and the sidelobes. The details are discussed in Choudhuri et al. 2014, 2016. The tapering is introduced by multiplying the sky with a window function. $\mathcal{W}(\theta)=e^{-\theta^{2} / \theta_{w}^{2}}$. Here, we implement the tapering by convolving the measured visibilities with Fourier transform of the window function. We have used the TGE to estimate $C_{\ell}$ and its variance in bins of equal logarithmic interval in $\ell$. In this work we correct each baseline with proper frequency scaling and finally collapse all channels to estimate the $C_{\ell}$.

$\dagger$ http://tgss.ncra.tifr.res.in 

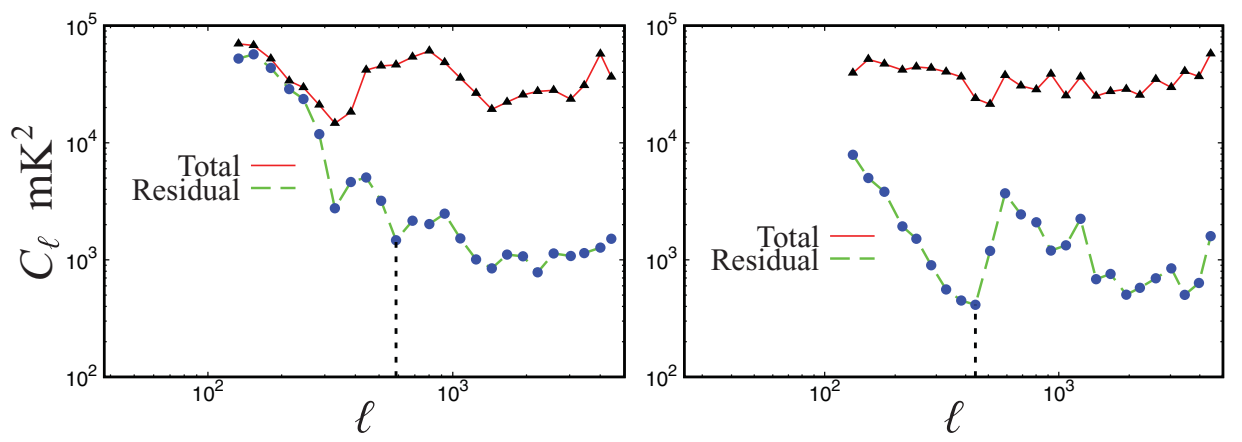

Figure 1. The estimated $C_{\ell}$ for Data1 and Data2 are shown in the left and right panel respectively. The upper and lowers curves are for before and after suntracting the point sources respectively. We identify a region shown by the vertical dotted lines in both panels $\left(\ell \geqslant \ell_{\max }\right)$ after which the residual $C_{\ell}$ is dominated by unsubtracted point sources. This figure is taken from Choudhuri et al. 2017.
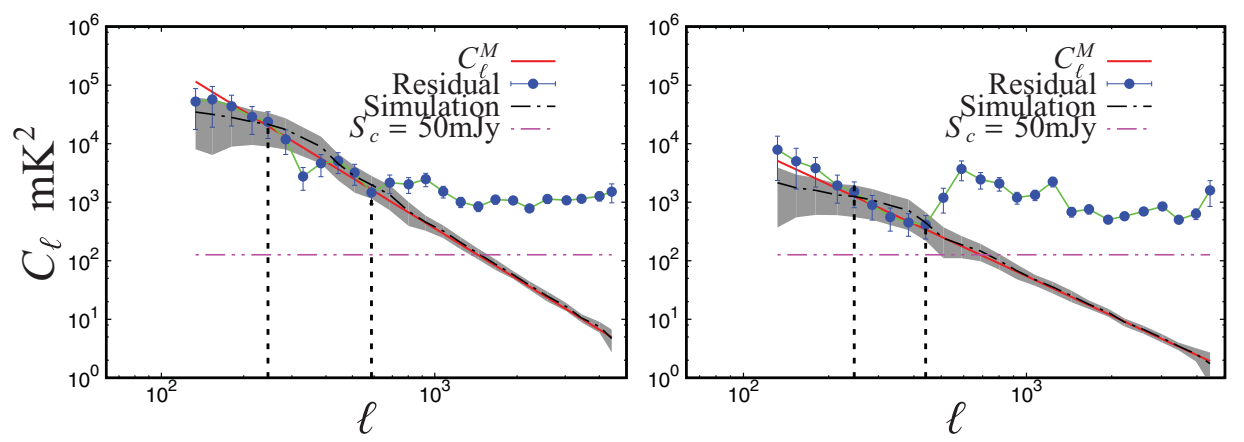

Figure 2. The estimated $C_{\ell}$ from the residual data (solid circles) with $1 \sigma$ error bar for Data1 is shown in the left panel. Two vertical lines are to show the region where the DGSE is expected to be dominated. The $C_{\ell}$ using the best fit parameters is shown by the solid line. The simulated $C_{\ell}$ using the best fit parametes is shown by the dash-dot line and the corresponding $1-\sigma$ error is shown with shaded region. The theoretical prediction for the $C_{\ell}$ due to residual point sources upto flux density $S_{c}=50 \mathrm{mJy}$ is shown by dot-dot-dash line. The right panel shows the same but for Data2. This figure is taken from Choudhuri et al. 2017.

\section{Results and Conclusions}

Figure 1 shows the estimated $C_{\ell}$ before and after subtracting the point sources from the central region of the primary beam. The left and right panels of Figure 1 are for Data1 and Data2 respectively. The upper curves in this figure show the $C_{\ell}$ before subtracting any point sources from the data. For both data sets the measured $C_{\ell}$ is in the range $10^{4}-10^{5} \mathrm{mK}^{2}$ for all values of $\ell$. The estimated $C_{\ell}$ is mainly dominated by the combination of the point sources and diffuse Galactic synchrotron emission. The lower curves in this figure show the measured $C_{\ell}$ after subtracting the point sources from the data. we subtract all the point sources upto $5 \sigma_{n}$ cut off level from the central region of the FoV. The estimated $C_{\ell}$ in lower $\ell$ values are affected by the convolution primary beam (Figure 3, Choudhuri et al. 2014). At large $\ell$, the residual $C_{\ell}$ is mainly dominated by the unsubtracted point sources. We identify a region in the $\ell$ space $\ell \leqslant \ell_{\max }\left(\ell_{\max }=580\right.$ and 440 for Data1 and Data2 respectively) where we believe the diffuse Galactic synchrotron emission has a significant contribution. The left and right panels of Figure 2 show the residual $C_{\ell}$ with $1-\sigma$ error bar for Data1 and Data2 respectively. Two vertical lines in these figures show the region $\left(\ell_{\min }, \ell_{\max }\right)$ where we expect the estimated $C_{\ell}$ is likely 
dominated by the Galactic synchrotron emission. The estimated $C_{\ell}$ behaves as a power law in this region. We fit a power law, $C_{\ell}=A \times\left(\frac{1000}{l}\right)^{\beta} \mathrm{mK}^{2}$ to the measured $C_{\ell}$ in this $\ell$ range. The best fits values of $(A, \beta)$ are $(356.23 \pm 109.5,2.8 \pm 0.3)$ and $(54.6 \pm 26,2.2 \pm 0.4)$ for Data1 and Data2 respectively. The values of $\beta$ from this analysis are quite consistent with earlier measurements (Bernardi et al. 2009; Ghosh et al. 2012; Iacobelli et al. 2013). In Figure 2, we have also shown the $C_{\ell}$ using the simulated data. In this simulation, we have used best-fit values of $A$ and $\beta$. The $1-\sigma$ errors for the simulated $C_{\ell}$, estimated using 128 independent realizations, are also shown by a shaded region. We have shown the theoretical prediction for the $C_{\ell}$ due to residual point sources in a situation where the all bright sources of flux density $S>50$ mJy has been subtracted from the data. In our analysis, the measured $C_{\ell}$ at $\ell>\ell_{\max }$ is somewhat overestimated as compared with the theoretical prediction. It may be due to the error in modelling and subtracting point sources from the central region.

In summary, we have estimated the angular power spectrum $C_{\ell}$ using two fields observed by TGSS in the $\ell$ range $150 \leqslant \ell \leqslant 4000$. We identify the region in $\ell$ space $(240 \leqslant \ell \lesssim 500)$ which we expect to be dominated by the DGSE. We present a power law fits to the estimated $C_{\ell}$ over this $\ell$ range. The best fit values of the amplitude $(A)$ and the power law index $(\beta)$ are $(356.23 \pm 109.5,2.8 \pm 0.3)$ and $(54.6 \pm 26,2.2 \pm 0.4)$ for two data sets considered here. The values of $\beta$ are consistent with the earlier measurements. We plan to extend this analysis for the whole sky and study the variation of the amplitude $(A)$ and power law index $(\beta)$ of $C_{\ell}$ using the full TGGS survey in future.

\section{Acknowledgements}

S. Choudhuri would like to acknowledge the University Grant Commission, India for providing financial support. S. Choudhuri would also like to thank the organizers of the IAUS 333 conference.

\section{References}

Ali, S. S., Bharadwaj, S., \& Chengalur, J. N., 2008, MNRAS, 385, 2166

Bernardi, G. et al. 2009, AAP, 500, 965

Bharadwaj, S. \& Ali, S. S., 2005, MNRAS, 356, 1519

Bowman, J. D. et al. 2013, Pub. Astro. Soc. Australia, 30, 31

Choudhuri, S., Bharadwaj, S., Ali, S. S., Roy, N., Intema, H. T., \& Ghosh A., 2017, MNRAS, 470, L11

Choudhuri, S., Bharadwaj, S., Chatterjee, S., Ali, S. S., Roy, N., \& Ghosh A., 2016, MNRAS, 463,4093

Choudhuri, S., Bharadwaj, S., Ghosh, A., \& Ali, S. S., 2014, MNRAS, 445, 4351

Dowell, J., Taylor, G. B., Schinzel, F. K., Kassim, N. E., \& Stovall, K., 2017, MNRAS, 469, 4537

Ghosh, A., Prasad, J., Bharadwaj, S., Ali, S. S., \& Chengalur, J. N., 2012, MNRAS, 426, 3295

Guzmán A. E., May, J., Alvarez, H., \& Maeda, K., 2011, AAP, 525, A138

Haslam, C. G. T., Salter, C. J., Stoffel, H., \& Wilson, W. E., 1982, AAPS, 47, 1

Iacobelli, M. et al. 2013, AAP, 558, A72

Intema, H. T., 2014, in Astronomical Society of India Conference Series, Vol. 13, Astronomical Society of India Conference Series

Intema, H. T., Jagannathan, P., Mooley, K. P., \& Frail, D. A., 2017, AAP, 598, A78

Intema, H. T., van der Tol S., Cotton, W. D., Cohen, A. S., van Bemmel I. M., \& Röttgering H. J. A., 2009, AAP, 501, 1185

Koopmans, L. et al. 2015, Advancing Astrophysics with the Square Kilometre Array (AASKA14), 1

Lazarian, A. \& Pogosyan, D., 2012, ApJ, 747, 5 
Mellema, G. et al. 2013, Experimental Astronomy, 36, 235

Neben, A. R. et al. 2016, ApJ, 826, 199

Parsons, A. R. et al. 2010, AJ, 139, 1468

Pritchard, J. R. \& Loeb, A., 2012, Reports on Progress in Physics, 75, 086901

Reich, P. \& Reich, W., 1988, AAPS, 74, 7

Reich, W., 1982, AAPS, 48, 219

Santos, M. G., Cooray, A., \& Knox, L., 2005, ApJ, 625, 575

Shaver, P. A., Windhorst, R. A., Madau, P., \& de Bruyn A. G., 1999, AAP, 345, 380

Sirothia S. K., Lecavelier des Etangs A., Gopal-Krishna, Kantharia N. G., \& Ishwar-Chandra C. H., 2014, AAP, 562, A108

Tingay, S. J. et al. 2013, Pub. Astro. Soc. Australia, 30, 7

van Haarlem, M. P. et al. 2013, AAP, 556, A2

Waelkens, A. H., Schekochihin, A. A., \& Enßlin T. A., 2009, MNRAS, 398, 1970

Yatawatta, S. et al. 2013, AAP, 550, A136 